JOURNAL OF THE

CHUNGCHEONG MATHEMATICAL SOCIETY

Volume 26, No. 2, May 2013

\title{
PORTFOLIO CHOICE UNDER INFLATION RISK: MARTINGALE APPROACH
}

\author{
ByUng HwA Lim*
}

\begin{abstract}
The optimal portfolio selection problem under inflation risk is considered in this paper. There are three assets the economic agent can invest, which are a risk free bond, an index bond and a risky asset. By applying the martingale method, the optimal consumption rate and the optimal portfolios for each asset are obtained explicitly.
\end{abstract}

\section{Introduction}

The inflation risk affects the financial investment, especially bond trading. In spite of the importance of considering the inflation risk on investment, there are not much attention to incorporate the risk into financial activities such as portfolio choice. The most difficult part is the fact that the inflation risk is non-hedgeable risk in real world. Although the inflation risk is undiversifiable, Kothari and Shanken [4] shows that the index bond could be the instrument to hedge the risk so we can make the financial market complete by introducing the index bond in market.

We attack Merton's model $[5,6]$ under inflation risk where there are risk free bond, index bond, and risky assets in market. Differently from Brennan and Xia [1], Munk et al. [7] and Gong and Li [2], we apply the martingale approach to get the value function and the optimal values. Gong and $\mathrm{Li}[2]$ uses DPP(dynamic programming principle) to get the value function for classical merton's problem under inflation risk and subsistence constraints. Their uncertainty sources from risky asset and inflation risk, however, are same so it is possible to apply DPP with one state variable. Brennan and Xia [1] and Munk et al.[7] consider the

Received January 18, 2013; Accepted April 04, 2013.

2010 Mathematics Subject Classification: Primary 91G10.

Key words and phrases: inflation risk, CRRA utility, asset allocation. 
portfolio selection problem in stochastic environment including inflation risk and also apply DPP to derive the optimal policies.

This paper is organized as follows. Section 2 and Section 3 explain a financial market setup and objective function we want to maximize by choosing consumption and investment. Section 4 gives the optimal policies and value function explicitly.

\section{Financial market}

There are three kinds of assets in the financial market, which are risk free bond, index bond, and risky asset. The risk free rate is a nominal interest rate given by $R>0$ and the risky asset follows the geometric Brownian motion with constant drift $\mu_{s}$ and constant volatility $\sigma_{s}$. Furthermore, the price process is also supposed to evolve

$$
\frac{d P_{t}}{P_{t}}=\mu_{p} d t+\sigma_{p} d W_{t}^{1}
$$

where $\mu_{p}$ and $\sigma_{p}$ are constant coefficients and $W_{t}^{1}$ is the standard Brownian motion under the probability space $(\Omega, \mathcal{F}, \mathbb{P})$. Then the risk free bond $B_{t}$, index bond $S_{t}^{0}$, and risky asset $S_{t}$ evolve respectively

$$
\frac{d B_{t}}{B_{t}}=R d t, \quad \frac{d S_{t}^{0}}{S_{t}^{0}}=r d t+\frac{d P_{t}}{P_{t}}, \quad \frac{d S_{t}}{S_{t}}=\mu_{s} d t+\sigma_{s} d W_{t}^{2},
$$

where $r$ is a constant real interest rate and $W_{t}^{2}$ is also the standard Brownian motion under the measure $\mathbb{P}$, which is independent of $W_{t}^{1} \cdot{ }^{1}$

Let's denote a feasible consumption rate and the portfolio ratios for each asset by $c_{t}, \pi_{t}^{0}, \pi_{t}^{1}$ and $\pi_{t}^{2}$ respectively. Furthermore, they are assumed to be $\mathcal{F}_{t}$-progressively measurable and satisfy

$$
\int_{0}^{\infty} c_{t} d t<\infty, \quad \quad \int_{0}^{\infty} \pi_{t}^{i^{2}} d t<\infty, \quad i=0,1,2 .
$$

Then the nominal wealth process $\bar{X}_{t}$ is unfolded by

$$
d \bar{X}_{t}=\pi_{t}^{0} \bar{X}_{t} \frac{d B_{t}}{B_{t}}+\pi_{t}^{1} \bar{X}_{t} \frac{d S_{t}^{0}}{S_{t}^{0}}+\pi_{t}^{2} \bar{X}_{t} \frac{d S_{t}}{S_{t}}-\bar{c}_{t} d t, \quad X_{0}=x
$$

and the inflation adjusted wealth process $X_{t}$ is characterized by

\footnotetext{
${ }^{1}$ It can be assumed that the Brownian motions $W_{t}^{1}$ and $W_{t}^{2}$ are correlated with certain correlation. The correlated Brownian motion can be decomposed by two independent Brownian motions, however so that our method can also be applied.
} 
$(2.1)$

$$
\begin{aligned}
& d X_{t} \\
& =d\left(\bar{X}_{t} / P_{t}\right) \\
& \begin{aligned}
= & \left.\left(\pi_{t}^{0}+\pi_{t}^{2}\right)\left(r+\mu_{p}-R-\sigma_{p}^{2}\right)+\pi_{t}^{2}\left(\mu_{s}-R\right)\right] X_{t} d t-c_{t} d t \\
& \quad+\pi_{t}^{2} X_{t} \sigma_{t} d W_{t}^{2}-\left(\pi_{t}^{0}+\pi_{t}^{2}\right) X_{t} \sigma_{p} d W_{t}^{1}, \quad X_{0}=x .
\end{aligned}
\end{aligned}
$$

To make the dynamic wealth process as static constraint, we introduce the equivalent martingale measure $\mathbb{Q}$ generated from the following exponential martingale process

$$
\xi_{t}=e^{-\frac{1}{2} \int_{0}^{t}\left(\theta_{1}^{2}+\theta_{2}^{2}\right) d s-\int_{0}^{t} \theta_{1} d W_{s}^{2}-\int_{0}^{t} \theta_{2} d W_{s}^{1}},
$$

where $\theta_{1}=\sigma_{s}^{-1}\left(\mu_{s}-R\right)$ and $\theta_{2}=\sigma_{p}^{-1}\left(r+\mu_{p}-R-\sigma_{p}^{2}\right)$. Under the measure $\mathbb{Q}$, the standard Brownian motions are redefined by,

$d \tilde{W}_{t}^{2}=d W_{t}^{2}+\sigma_{s}^{-1}\left(\mu_{s}-R\right) d t, \quad d \tilde{W}_{t}^{1}=d W_{t}^{1}+\sigma_{p}^{-1}\left(r+\mu_{p}-R-\sigma_{p}^{2}\right) d t$.

Therefore, under the new measure $\mathbb{Q}$ the inflation risk adjusted wealth process becomes

$$
d X_{t}=\left[r X_{t}-c_{t}\right] d t+\pi_{t}^{2} X_{t} \sigma_{s} d \tilde{W}_{t}^{2}-\left(\pi_{t}^{0}+\pi_{t}^{2}\right) X_{t} \sigma_{p} d \tilde{W}_{t}^{1} .
$$

We can verify that under the equivalent martingale measure, the risk adjusted wealth process is the wealth process with real interest rate only. In other words, the real interest rate is the only factor for saving under the new measure. If we define the pricing kernel as $H_{t}=e^{-r t} \xi_{t}$, it is well-known that the wealth dynamics (2.1) is transformed to

$$
\mathbb{E}\left[\int_{0}^{\infty} H_{t} c_{t} d t\right] \leq x .
$$

\section{Optimization problem}

Our object function is the expected utility maximization by choosing consumption rate and portfolio ratios. In this paper, the agent's preference is supposed to be defined by CRRA(constant relative risk aversion) utility function. With a risk aversion $\gamma(\gamma>0, \gamma \neq 1)$, the utility function is expressed as $u\left(c_{t}\right)=\frac{1}{1-\gamma} c_{t}^{1-\gamma}$.

So the agent's expected utility maximization problem with discount factor $\beta$ is represented by

$$
\max _{c, \pi} \mathbb{E}\left[\int_{0}^{\infty} e^{-\beta t} u\left(c_{t}\right) d t\right]
$$


subject to the wealth constraint (2.2). The agent's optimal choice variables are consumption rate and investments for index bond and risky assets. Then the savings are the remaining wealth after investing and consumption.

To apply the duality approach, we introduce the Lagrange multiplier $\lambda>0$ so that the dual value function is defined by

$$
J(\lambda)=\max _{c, \pi}\left\{\mathbb{E}\left[\int_{0}^{\infty} e^{-\beta t} u\left(c_{t}\right) d t\right]-\lambda \mathbb{E}\left[\int_{0}^{\infty} H_{t} c_{t} d t\right]\right\} .
$$

Since the CRRA utility function is concave and non-decreasing and has derivative function which is continuous, positive, and strictly decreasing, there exists the continuous inverse function $I:(0, \infty) \rightarrow(0, \infty)$ of $u^{\prime}(\cdot)$. Then the convex dual of a utility function $u(\cdot)$ is the function

$$
\widetilde{u}(y)=\sup _{x \in \mathbb{R}}\{u(x)-x y\}, \quad y \in \mathbb{R},
$$

and it is easily checked that

$$
\widetilde{u}(y)=u(I(y))-y I(y), \quad y>0,
$$

where $I(y)=y^{-\frac{1}{\gamma}}$ and $\widetilde{u}(y)=\frac{\gamma}{1-\gamma} y^{-\frac{1-\gamma}{\gamma}}$ for the CRRA utility function. The optimal consumption rate is determined while constructing the dual value function and the inverse function is directly related to the optimal consumption rate.

If we confirm the dual value function, the value function of the original problem is given by the relation obtained from finding the minimizing multiplier $\lambda$.

Lemma 3.1. When the dual value function is given by the equation in (3.2), the value function of the primal problem is determined by

$$
V(x)=\inf _{\lambda>0}\{J(\lambda)+\lambda x\} .
$$

Proof. See Karatzas and Shereve [4].

While finding the optimal $\lambda^{*}$, the optimal wealth is determined and its dynamics can also be characterized. Thus the optimal portfolios are given by comparing with the wealth dynamics in (2.1).

\section{Main results}

From the duality, the agent's maximization problem can be resolved by deriving the dual value function first. 
Proposition 4.1. The dual value function defined in (3.2) is obtained from

where

$$
J(\lambda)=\frac{\gamma}{K(1-\gamma)} \lambda^{-\frac{1-\gamma}{\gamma}}
$$

$$
K=r+\frac{\beta-r}{\gamma}-\frac{1-\gamma}{2 \gamma^{2}}\left(\theta_{1}^{2}+\theta_{2}^{2}\right)
$$

Proof. The dual value function is characterized by

$$
\begin{aligned}
J(\lambda) & =\max _{c, \pi} \mathbb{E}\left[\int_{0}^{\infty} e^{-\beta t}\left\{u\left(c_{t}\right)-\lambda e^{\beta t} H_{t} c_{t}\right\} d t\right] \\
& =\mathbb{E}\left[\int_{0}^{\infty} e^{-\beta t} \widetilde{u}\left(\lambda e^{\beta t} H_{t}\right) d t\right] \\
& =\mathbb{E}\left[\int_{0}^{\infty} e^{-\beta t} \frac{\gamma}{1-\gamma}\left(\lambda e^{\beta t} H_{t}\right)^{-\frac{1-\gamma}{\gamma}} d t\right] .
\end{aligned}
$$

The second equality comes from substituting the optimal consumption rate which is

$$
c_{t}^{*}=I\left(\lambda e^{\beta t} H_{t}\right)=\left(\lambda e^{\beta t} H_{t}\right)^{-\frac{1}{\gamma}},
$$

and the third equality is the case for CRRA utility function.

Let's denote the conditional expectation of the last equation at time $t$ by

$$
\phi(t, y)=\mathbb{E}_{t}\left[\int_{t}^{\infty} e^{-\beta s} \frac{\gamma}{1-\gamma}\left(y_{s}\right)^{-\frac{1-\gamma}{\gamma}} d s\right],
$$

where $y_{t} \triangleq \lambda e^{\beta t} H_{t}$ and has differential form of

$$
\frac{d y_{t}}{y_{t}}=(\beta-r) d t-\theta_{1} d W_{t}^{2}-\theta_{2} d W_{t}^{1} .
$$

Then by Fynmman-Kac's formula, the function $\phi(t, y)$ should satisfy PDE(partial differential equation) generated by $d y_{t}$

$$
\frac{\partial \phi}{\partial t}+\frac{\partial \phi}{\partial y} y(\beta-r)+\frac{1}{2} \frac{\partial^{2} \phi}{\partial y^{2}}\left(\theta_{1}^{2}+\theta_{2}^{2}\right)+e^{-\beta t} \frac{\gamma}{1-\gamma} y^{-\frac{1-\gamma}{\gamma}}=0 .
$$

If we suggest the separable solution as $\phi(t, y)=e^{-\beta t} v(y)$, the PDE is reduced to the ODE(ordinary differential equation) for $v(y)$ :

$$
\frac{1}{2} y^{2} v^{\prime \prime}(y)\left(\theta_{1}^{2}+\theta_{2}^{2}\right)+y v^{\prime}(y)-\beta v(y)+\frac{\gamma}{1-\gamma} y^{-\frac{1-\gamma}{\gamma}}=0 .
$$

From the variation of parameter, we can get the solution to the ODE. Since the solution function has a finite value for $y \in[0, \infty]$, the general 
solution part should be zero. Then the particular solution for ODE (4.3) is easily obtained by guessing $v(y)=A y^{-\frac{1-\gamma}{\gamma}}$.

If we substitute the function and its derivatives into the ODE (4.3), with the constant $K$ in (4.1) the coefficient $A$ is given by $A=\frac{\gamma}{K(1-\gamma)}$. Accordingly, the dual value function is derived by

$$
J(\lambda)=\phi(0, \lambda)=v(\lambda) .
$$

Theorem 4.2. The economic agent who faces the inflation risk has the value function for the expected utility maximization in (3.1) as

$$
V(x)=\frac{K^{-\gamma}}{(1-\gamma)} x^{1-\gamma},
$$

and for the agent's wealth $X_{t}$ the optimal consumption and portfolios are given by

$$
\begin{gathered}
c_{t}^{*}=K X_{t}, \\
\pi_{t}^{0^{*}}=-\left(\frac{\theta_{2}}{\sigma_{p} \gamma}+\frac{\theta_{1}}{\sigma_{s} \gamma}\right), \quad \pi_{t}^{1 *}=\left(\frac{\theta_{2}}{\sigma_{p} \gamma}+1\right), \quad \pi_{t}^{2^{*}}=\frac{\theta_{1}}{\sigma_{s} \gamma},
\end{gathered}
$$

where the process $y_{t}^{\lambda^{*}}$ should satisfy $X_{t}=\frac{1}{K}\left(y_{t}^{\lambda^{*}}\right)^{-\frac{1}{\gamma}}$.

Proof. The value function of the original problem is easily confirmed from Lemma 3.1 and Proposition 4.1. Furthermore, while we find the optimal $\lambda$ in Lemma 3.1, the optimal wealth process is induced by

$$
X_{t}^{*}=\frac{1}{K}\left(y_{t}^{\lambda^{*}}\right)^{-\frac{1}{\gamma}}
$$

So with the dynamics of $y_{t}^{\lambda^{*}}$ in (4.2), the optimal wealth process $X_{t}^{*}$ should be governed by

$$
\begin{aligned}
d X_{t}^{*}=\left[-\frac{1}{\gamma K}\left(y_{t}^{\lambda^{*}}\right)^{-\frac{1}{\gamma}}(\beta-r)+\frac{1}{2 \gamma^{2} K}\left(y_{t}^{\lambda^{*}}\right)^{-\frac{1}{\gamma}}\left(\theta_{1}^{2}+\theta_{2}^{2}\right)\right] d t \\
\quad+\frac{\theta_{1}}{\gamma K}\left(y_{t}^{\lambda^{*}}\right)^{-\frac{1}{\gamma}} d B_{t}+\frac{\theta_{2}}{\gamma K}\left(y_{t}^{\lambda^{*}}\right)^{-\frac{1}{\gamma}} d W_{t} \\
=\left[r+\pi_{t}^{2^{*}}\left(\mu_{s}-R\right)+\pi_{t}^{1^{*}}(r-R)-\left(\pi_{t}^{0^{*}}+\pi_{t}^{2^{*}}\right) \mu_{p}\right] X_{t}^{*} d t-c_{t}^{*} d t \\
\quad+\pi_{t}^{2^{*}} X_{t}^{*} \sigma d B_{t}-\left(\pi_{t}^{0^{*}}+\pi_{t}^{2^{*}}\right) X_{t}^{*} \sigma_{p} d W_{t}
\end{aligned}
$$

where the optimal policies are defined by

$$
c_{t}^{*}=\left(y_{t}^{\lambda^{*}}\right)^{-\frac{1}{\gamma}}, \quad \pi_{t}^{0^{*}} X_{t}^{*}=-\left(\frac{\theta_{2}}{\sigma_{p}}+\frac{\theta_{1}}{\sigma}\right)\left[\frac{1}{\gamma K}\left(y_{t}^{\lambda^{*}}\right)^{-\frac{1}{\gamma}}\right],
$$




$$
\pi_{t}^{1^{*}} X_{t}^{*}=\frac{\theta_{2}}{\sigma_{p}}\left[\frac{1}{\gamma K}\left(y_{t}^{\lambda^{*}}\right)^{-\frac{1}{\gamma}}\right]+X_{t}^{*}, \quad \pi_{t}^{2^{*}} X_{t}^{*}=\frac{\theta_{1}}{\sigma}\left[\frac{1}{\gamma K}\left(y_{t}^{\lambda^{*}}\right)^{-\frac{1}{\gamma}}\right] .
$$

By substituting the optimal wealth in (4.4) into each optimal policies, we get the results.

As we expected, when the nominal interest rate is lower than inflation adjusted real interest rate $\left(\theta_{2}=\sigma_{p}^{-1}\left(r+\mu_{p}-R-\sigma_{p}^{2}\right)>0\right)$, the agent borrows money from bank and invests index bond to hedge the risk.

\section{References}

[1] M. P. Brennan and Y. Xia, Dynamic Asset Allocation under Inflation, J. Financ. 57 (2002), 1201-1238.

[2] N. Guo and T. Li, Role of Index Bonds in an Optimal Dynamic Asset Allocation Model with Real Subsistence Consumption, Appl. Math. Comput. 174 (2006), $710-731$.

[3] S. P. Kothari and J. Shanken, Asset Allocation with Inflation-protected Bonds, Financ. Analysts Journal 60 (2004), 54-70.

[4] I. Karatzas and S. E. Shreve, Methods of Mathematical Finance, Springer, New York, 1998.

[5] R. C. Merton, Lifetime Portfolio Selection under Uncertainty: the ContinuousTime Case, Rev. Econ. Stat. 51 (1969), 247-257.

[6] R. C. Merton, Optimum Consumption and Portfolio Rules in a ContinuousTime Model, J. Econ. Theory 3 (1971), 373-413.

[7] C. Munk, C. Sørensen, and T. N. Vinther, Dynamic Asset Allocation under Mean-reverting Returns, Stochastic Interest rates, and Inflation Uncertainty: Are Popular Recommendations consistent with Rational Behavior?, Int. Rev. Econm. Financ. 13 (2004), 141-166.

Graduate School of Financial Engineering

The University of Suwon

Hwaseong 445-743, Republic of Korea

E-mail: byunghwalim@suwon.ac.kr 